\title{
A case of failed vertical augmentation of a post- traumatic mandibular defect by distraction osteogenesis
}

\begin{abstract}
One trauma case is presented. An ice-hockey puck fractured the mandible and caused major soft tissue damages including the periosteum. After healing the alveolar ridge was lost and a reconstruction had to be made. Distraction osteogenesis (DO) treatment was conducted but a postoperative infection disrupted the bone distraction phase and eventually synthetic bone matrix had to be used. The patient experienced considerable problems post-DO surgery. However, after three surgical procedures dental implants were inserted. DO is a reliable treatment in patients where vertical bone height is missing, and healthy tissue is present. However, when the periosteum is damaged or postoperative infection is present, DO is disturbed and in this case a synthetic bone matrix had to be employed. One and a half years later a prosthetic bridge has been installed giving delayed but satisfactory result.
\end{abstract}

Volume 6 Issue 4 - 2017

\author{
Annika Rosen,' Carina Kruger Weiner ${ }^{2,3}$ \\ 'Department of Clinical Dentistry-Section of Oral \& \\ Maxillofacial Surgery, University of Bergen, Norway \\ ${ }^{2}$ Department of Dental Medicine-Division of Oral Facia \\ Diagnostics and Surgery, Karolinska Institute, Sweden \\ ${ }^{3}$ Department of Oral and Maxillofacial Surgery, Eastman \\ Institute, Sweden
}

Correspondence: Annika Rosen, Department of Clinica Dentistry- Section of Oral \& Maxillofacial Surgery, Faculty of Medicine and Dentistry, University of Bergen, Norway, Tel +47 5558648I,Email annika.rosen@uib.no

Received:September 08, 2016 | Published: February 23, 2017

\section{Introduction}

Distraction osteogenesis (DO) is a method first employed in orthopaedic surgery for the lengthening of long bones. ${ }^{1}$ In the craniomaxillofacial area, the first case was presented more than 20 years ago. ${ }^{2}$ When the level of vertical alveolar bone is too low for dental implants DO can be used. ${ }^{3}$ In addition, DO has also been employed after trauma for restoring wounded jaws., ${ }^{4,5}$ The major advantage to DO is that a bone graft is not necessary, it also allows the soft tissue to expand into the oral region and it is comparatively a minor surgical procedure. ${ }^{6}$ Currently, the literature regarding vertical augmentation of resorbed ridges has presented some prospective clinical studies. ${ }^{7-9}$ Maxillofacial fractures and mandibular condylar fractures treated with DO have been presented in humans. Mandibular corpus fractures treated with DO have been shown to be a robust and quantifiable method that increases the vascular density compared with ordinary fracture healing in rats. ${ }^{10}$ Until now no reports have been presented in humans. Therefore, this article considers a review of the literature involving alveolar DO, a clinical patient case, critical surgical factors, and complications for oral rehabilitation of reconstructed jaws.

\section{Presentation of a trauma case}

A 35-year old male had been hit by a hockey puck on the right side of the mandible. It resulted in a mandible fracture producing major loss of the alveolar bone and loss of four teeth and soft tissue damage. The fracture was stabilized with titanium plates and a bi-cortical screw three days after trauma. The healing process following surgery was normal. However, after six months, radiological and clinical examinations showed a defect in height of the alveolar bone and loss of the firm mucosa. The alveolar ridge needed to be reconstructed before dental implants could be inserted and a jaw-bone anchored bridge put in place for optimal chewing function. The treatment plan was to use DO and distract the alveolar segment region 41-45. When the tissue flap was raised in the mandible it was discovered that very little of the periosteum remained and was damaged. The distractor was fixated and activated (Figure 1). Unfortunately, the mucosa opened up postoperatively and a smaller surgical procedure took place two weeks later, where the remaining periosteum was lifted up over the distraction device. During this time the patient was treated with antibiotics for postoperative infection. The retention phase lasted for six months, because of the postoperative infection. The patient was scheduled for regularly appointments at the clinic and the clinical stability of the device was controlled. Six months after treatment, a computer tomography (CT) scan showed a defect in bone healing around the distracted bone (Figure 2). In addition, during this period firm gingiva had developed over the lingual alveolar ridge and replaced the loose soft tissue that was found after initial trauma healing. We decided to open up the former surgery area to remove the DO device and explore the remaining alveolar bone. Examination of the alveolar crest revealed bone sequestered on the buccal side, and it was subsequently removed. It was apparent that the lingual alveolar ridge remained and approximately half the width of the crest had been successfully distracted but unfortunately it was too narrow for implant placement. A bone building therapy with a synthetic bone matrix was used (BioOss) to recover the full width of the bone. The bone matrix was packed against the lingual bone wall; a membrane held it together and finally the mucosal lap was lifted. The periosteum and the soft tissue were thoroughly sutured. One week after surgery, again the wound re-opened and the area was exposed intra-orally. After approximately one month of secondary healing the wound closed. Nine months after the re-healing period a radiological examination was made which showed true integration of the bovine bone material (Figure 3). Four dental implants were 
placed in the mandibular alveolar crest (Figure 4). The implants were successfully osseointegrated, abutments were placed after five months and a prosthetic bridge was put in place, which gave the patient a functional occlusion.

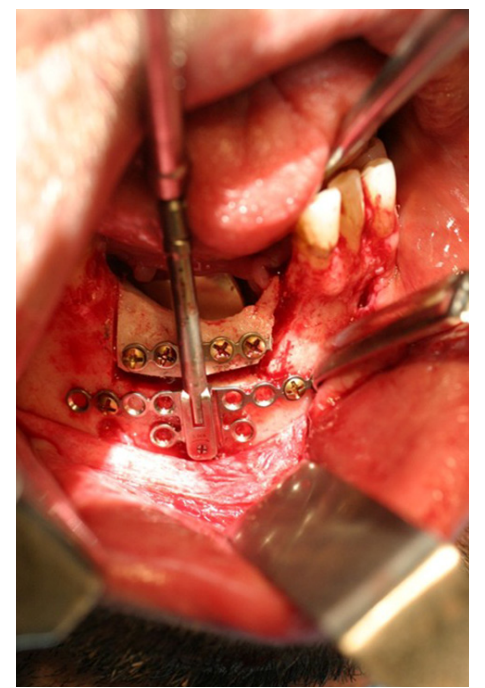

Figure I Intraoral picture showing the DO device put in place in the mandible for gaining vertical alveolar bone height.

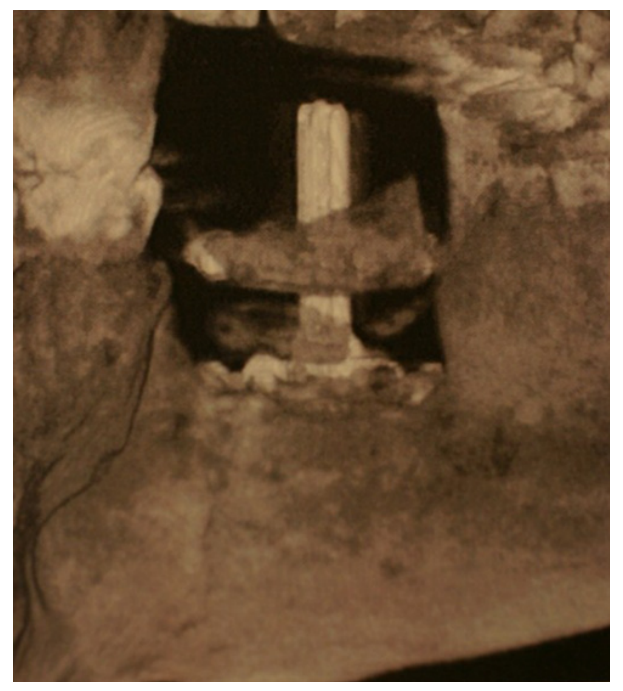

Figure 2 Result after DO treatment a CT scan showing necrotic bone.

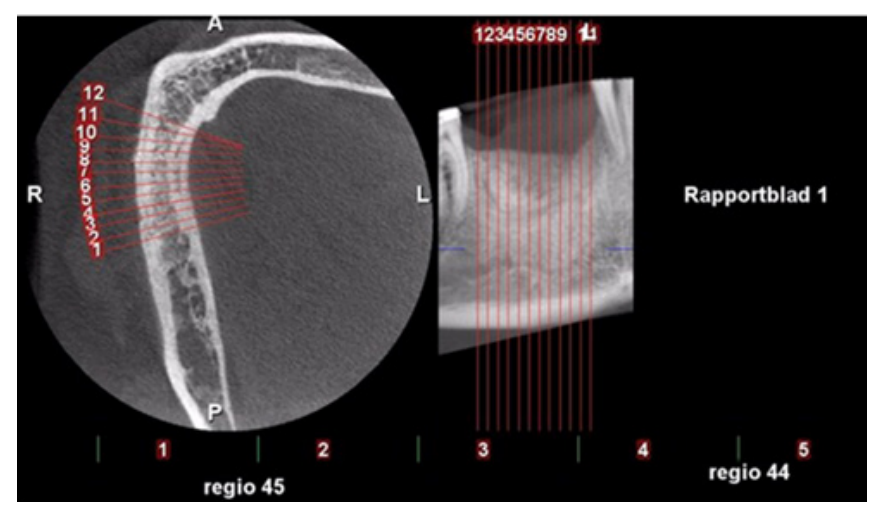

Figure 3 A CT scan showing vertical alveolar bone gained.

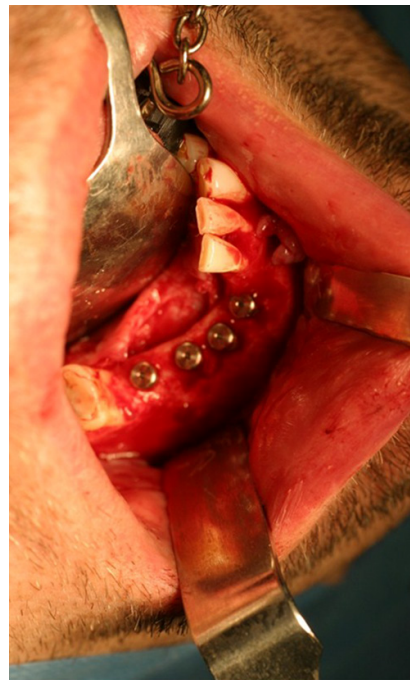

Figure 4 Intraoral picture showing four dental implants installed in the mandible, one year after DO.

\section{Discussion}

A trauma case is presented a 35 year old male hockey player who had sustained a mandibular fracture resulting in lost alveolar height. The patient was treated with DO but exhibited side effects, including a postoperative infection that delayed the reparative process. We highlight the importance of healthy tissue for successful outcome of DO treatment. In this patient, several surgical procedures had to be performed, DO treatment, bone building therapy with bovine bone and finally implant treatment were needed. However, a good result, although delayed was obtained, and recently a prosthetic bridge was installed. It is interesting to examine this patient in more detail, where as the result of a mandibular fracture, the whole part of the alveolar ridge was lost including the teeth in the area. Several factors can be the reason for disturbed healing of the tissue. One can speculate in that the disturbed healing was due to lack of vascularization in the damaged periosteum and a postoperative infection which exposed the bone to the oral microflora. Reasons for the postoperative infection could be due to the damaged periosteum, diminished vascularity/ blood supply because of the scar, and you could not exclude that the fixation of the DO-device was inadequately rigid in the first place. From a biological perspective the regenerative process seemed to be hindered during bone formation affected by the trauma and surgical treatments. A prerequisite for bone regeneration following trauma or postoperative repair is the development of an inflammatory response and the need for wound healing. In this patient case the alveolar ridge was resorbed during the healing period, probably because of the damaged periosteum and the postoperative infection that prevented proper wound healing. However, it was apparent that the lingual alveolar ridge remained and approximately half the width of the crest was successfully distracted, including firm gingiva that had developed over the alveolar ridge. Firm gingiva is a key structure when dental implants are installed. Surrounding firm gingiva at the implant site prevent future hygiene deficits which can develop to peri implantitis. Each time a surgical procedure is performed, an inflammation process is conducted. The wound has to be healed but wound healing is a complex process. Blood clots that are formed follow by infiltration of inflammatory cells hours after damage, and cleanses the wound of bacteria and necrotic debris. If the decontamination fails, proinflammatory cytokines may remain at elevated levels. ${ }^{11}$ The late 
phase of inflammation, assist the formation of granulation tissue and release growth factors that stimulate fibroblasts. Granulation tissue is rich in cells and the collagen matrix provides a suitable environment for cell proliferation and increase the vascularisation prior to bone formation. ${ }^{12}$ However, if pro-inflammatory cytokines continue to be expressed, the inflammatory period can be extended, as was the case for the patient with several surgical procedures. The patient required a second surgical intervention to remove accumulated granulation tissue, when the wound had entered a chronic state and failed to heal. Primary bone forms rapidly, particularly after fracture healing or during distraction of the bone. But if they DO device is not properly fixed to both bone ends, or will lose its contact to the bone, an inflammation process will start which will delay the bone remodelling phase. Several interesting points can be pointed out when considering the problems experienced by this patient. The patient's periosteum, that has been pointed out to be the key for bone regeneration, ${ }^{13}$ had been destroyed during trauma and a postoperative infection arose which led to the situation that he consequently missed a potential source of regenerative cells.

\section{Conclusion}

DO is a reliable treatment in patients where vertical bone height is missing, and healthy tissue is present. However, in cases where the periosteum is damaged and/or a postoperative infection is present precautions have to be taken into account when DO is performed.

\section{Consent}

Patient's written consent has been obtained to publish clinical photographs.

\section{Funding}

None.

\section{Acknowledgements}

The authors thank the Karolinska Institutet's funds and the Department of Clinical Dentistry, University of Bergen for the support of the study.

\section{Conflicts of interest}

The authors declare that there is no conflict of interest.

\section{References}

1. Ilizarov GL. Clinical application of the tension-stress effect of limb lengthening. Clinical Orthop Rel Res. 1990;250:8-26.

2. McCarthy JG, Schreiber J, Karp N, et al. Lengthening the human mandible by gradual distraction. Plast Reconstr Surg. 1992; 89(1):1-8.

3. Saulacic N, Iizuka T, Martin M, et al. Alveolar distraction osteogenesis: a systemic review. Int J Oral Maxillofac Surg. 2008; 37(1):1-7.

4. Cano J, Campo J, Moreno L, et al. Osteogenic alveolar distraction. A review of the literature. Oral Surg Oral Med Oral Pathol Oradiol Endod. 2006;101(1):11-28.

5. Cheung LK, Chua H, Hariri F, et al. Distraction osteogenesis. In: Oral and maxillofacial surgery. Andersson L \& Kahnberg KE, editors. Wileyblackwell, USA; 2010;48:1027-1059.

6. Diner PA, Kollar E, Marinez H, et al. Submerged intraoral device for mandibular lengthening. J Craniomaxillofac Surg. 1997;25(3):116-123.

7. Chiapasco M, Consolo U, Bianchi A, et al. Alveolar distraction osteogenesis for the correction of vertically deficient edentulous ridges. A multicenter prospective study on humans. Int J Oral Maxillofac implants. 2004;19(3):399-407.

8. Jensen OT, Cockrell R, Kuhike L, et al. Anterior maxillary alveolar distraction osteogenesis: a prospective 5-years clinical study. Int J Oral Maxillofac Implants. 2002;17(1):52-68.

9. Polo W, Cury PR, Sendyk WR, et al. Posterior mandibular distraction osteogenesis utilizing an extra-osseous distractor: a prospective study. $J$ Periodontol. 2005;76(9):1463-1468.

10. Donneys A, Tchanque Fossuo $\mathrm{CN}$, et al. Bone regeneration in distraction osteogenesis demonstrates significantly increased vascularity in comparison to fracture repair in the mandible. J Craniofac Surg. 2012;23(1):328-332.

11. Guo S, Dipietro LA. Factors that affecting wound healing. $J$ Dent Res. 2010;89(3):219-229.

12. Terheyden H, Lang NP, Bierbaum S, et al. Osseointegrationcommunication of cells. Clin Oral Implants Res. 2012;23(10): 11271135 .

13. Mahayan A. Periosteum: a highly underrated tool in dentistry. Int J Dent. 2012:717816. 